\title{
Principles of Natural Resource Economics for Bitcoin
}

\author{
Prateek Goorha
}

Bridgewater State University, Massachusetts, USA

\section{Correspondence: goorha@sent.com}

Received: 16 March 2021 Accepted: 23 June 2021 Published: 4 August 2021

\begin{abstract}
To assess claims such as Bitcoin is 'digital gold' it makes sense to examine whether Bitcoin exhibits features common to other exhaustible natural resources that are the concern of natural resource economists. We therefore present some foundational ideas in the economics of exhaustible resources and examine their relevance to Bitcoin. There are several useful similarities but also some key differences, chiefly with respect to how miners manage inventories, or their 'inventory policy'. Therefore, to highlight this aspect, we use a simple model for any physical natural resource and introduce sensitivity to a capital-to-energy ratio. The resulting policy for Bitcoin miner over a halving cycle is not unlike a traditional miner in that optimal inventories are determined by optimal capital investments over the entire duration of the cycle.
\end{abstract}

Keywords: Bitcoin, miner, exhaustible resources, inventory policy

JEL Classifications: Q3, D21 and G31

\section{Resource Economics and Bitcoin}

The claim that Bitcoin is 'digital gold' rests first and foremost on the soundness of the idea that Bitcoin behaves like a traditional exhaustible resource ${ }^{1}$; that it is more akin to gold than any other depletable resource relies further on the premise that it is relatively scarcer than gold. To that end, it is significant that Bitcoin has been programmed to mimic the essential characteristics of an exhaustible resource: its extraction rate approaches 0 over time and the total yield feasible from 'mining' is limited to 21 million bitcoins. It is, therefore, worth examining what analytical value the economics of exhaustible resources provides for the case of Bitcoin.

The economics of exhaustible resources has one rather simple and compelling analytical premise. It is that the opportunity costs incurred from current extraction and consumption of an exhaustible resource must be weighed against the fact that limited supplies ought to generate returns over time. A miner must, therefore, consider both the market value of a resource and the opportunity costs of current extraction in its investment decisions. The price of any such depletable resource should exceed its marginal cost of extraction to capture any logic of an exhaustible resource

\footnotetext{
${ }^{1}$ See [1] for a recent comparison of Bitcoin (specifically, price and hashrate behaviour) in terms of established results in energy economics regarding the oil and gas industry.
}

being used in the present rather than the future, the 'excess' representing the opportunity cost of intertemporal substitution in consumption.

While the market value of the natural stock of an unextracted resource depends on the prevailing market price and the attendant costs of bringing the resource to the market, the opportunity costs depend on trading off future consumption possibilities with present use as well as considerations on the present value of the rent that is destroyed by extracting in the present rather than leaving the resource in situ for the future.

The difference between marginal extraction costs and the price is often called the Hotelling rent in recognition of Hotelling's seminal 1931 paper [2]. It further follows from the preceding observations that the rate of change in price of the depletable resource must equal the interest rate that a miner uses to discount the future, and this is known as the Hotelling $r$-percent growth rule. Whenever marginal extraction costs are zero, the price of the resource in stock and that of the unmined resource are equivalent and the Hotelling rule applies equally to both. If, however, extraction costs increase over time, the price of the resource rises at less than the discount interest.

Thus, all things being equal, an increase in the discount rate implies a higher price for the unextracted resource and would incentivise a faster rate of extraction. In Bitcoin, 
while the rate of extraction is algorithmically fixed for any given halving cycle, mining effort can readily be increased. Further, miners arguably discount hyperbolically for the simple reason that the total remaining in situ stock is known in advance as well as the fact that extraction costs are likely to rise exponentially into the future as all miners increase efforts.

A relationship between Bitcoin and the economics of natural resources has been examined in a few other papers. [3] presents a continuous-time model for the inventory policy of miners in Bitcoin that permits examining how miners optimise over the income generated from transaction fees, while also accounting for risks that emanate from demandside shocks. By contrast, the ambition of this chapter is to present a simple model that places emphasis on highlighting the parallels in Bitcoin with natural resource mining and examines the inventory policy of Bitcoin miners over the course of a halving cycle. In this ambition, a notable contribution is the empirical analysis presented in [4]. The authors discuss the importance of the Hotelling rule to natural resource and energy economics, provide a useful review of the literature and, using Bitcoin as a case study, show strong support in the data that mining rents in Bitcoin are associated with the market rate of return.

\subsection{Some Slight Differences}

The Hotelling $r$-percent growth rule is sensitive to several factors in practice that have useful analogies in Bitcoin. ${ }^{2,3}$ These include: (a) the marginal cost incurred by the miner in exploration and extraction of the resource, which in the case of Bitcoin depends on the network difficulty and the requisite hashrate; (b) the perceived scarcity of the resource, i.e. the point on Bitcoin's overall trajectory of bitcoins mined relative to its absolute cap or total depletion; (c) the level of competition in mining, or, for Bitcoin, the relative hashrate of a miner to the overall hashrate being deployed by other miners across the network.

Being a digital resource, the size of the resource 'field' to be explored in Bitcoin over time can, in theory, increase or decrease in proportion with the exploratory effort of the miners. The miners deploy the hashpower of their mining rigs in order to increase the probability that their efforts to solve the cryptographic problem ${ }^{5}$ are successful, thereby earning them the right to add their block to the Bitcoin blockchain and receive the block reward. The more hashpower that is brought to bear across the network, the

\footnotetext{
${ }^{2}$ See [5] for a useful review of the literature inspired by Hotelling.

${ }^{3}$ We assume a basic familiarity Bitcoin; for the uninitiated, we suggest reading [6] and the literature cited in that paper.

${ }^{4}$ In this regard, [7] shows that the marginal cost of mining provides a strong support for the price of Bitcoin, making the analogy to natural resources stronger and to a pure speculative asset weaker.

${ }^{5}$ Recall that this involves using the SHA-256 hashing function twice, compressing arbitrary sized inputs into a fixed-length output in the process.
}

higher the difficulty ${ }^{6}$ of the cryptographic problem and vice versa, essentially adjusting the size of the resource field for miners based directly on their efforts. This isn't very different from any exhaustible natural resource that has increasing extraction costs over the long run with periods of falling costs that eventuate from new discoveries of resource sites or cost-saving technologies.

In contrast with any other natural resource, Bitcoin's exhaustion trajectory is far more deterministic. Many of the other features immanent to Bitcoin are, as a matter of fact, similar in nature to the assumptions made by Hotelling [2] in that Bitcoin mining is competitive, ${ }^{7}$ the overall stock of bitcoins is known exactly and that, while technology for mining does improve, it does so in lock-step with the algorithmically adjusted difficulty for mining. Thus, the Hotelling rule ought to provide at least a useful starting point for the case of Bitcoin.

As opposed to exhaustible natural resources, where the Hotelling rent depends on whether the resource is left in situ, decisions on timing when to mine bitcoin, however, isn't a free variable. While miners frequently do pool their resources into one of several larger mining pools to maximise their chances of finding a block, in general, mining cannot be unitised in Bitcoin in any meaningful way. Thus, bitcoins are mined competitively and with a strictly decreasing yield over time - a block reward that halves roughly every four years. Further, with Bitcoin, there is no possibility of unexpected discoveries or new technologies making currently inaccessible reservoirs of resources suddenly available for exploitation.

\subsection{And a Key Point of Difference}

It is well established that excessive competition in the rapid exploitation of a resource leads to social waste; a common property problem of restricting access drives the familiar tragedy of the commons. Bitcoin, however, expressly relies on and exploits the incentives that create the common property problem. While the mining of bitcoins is governed by an algorithmic mechanism that encourages 'excessive' competition, the value of the resulting waste that accrues from this mad rush of mining is internalised to the security of the Bitcoin network, since higher network hashrates directly result in commensurate difficulty increases. ${ }^{8}$

\footnotetext{
${ }^{6}$ Recall that the difficulty is adjusted by the Bitcoin code for every 2016 blocks, based upon whether the hashpower deployed over the network is trailing or leading a target of 10 -minutes per block.

7 The intuition is relatively straightforward. When an exhaustible resource is mined under conditions of monopoly, it will be extracted at a more gradual pace and price will remain above the marginal rate of extraction as it grows at a relatively more stable rate. Conversely, when the same resource is mined competitively, the rate of extraction will be higher, over a shorter horizon, and the price will grow faster over the entire period.

${ }^{8}$ This is not to say that, just like any other natural resource that is competitively exploited under rules of free access, mining in Bitcoin does not create social costs. Rather, the point is that competitive extraction creates an externality on network security in Bitcoin, which is an effect not seen with physical natural resources.
} 
Since bitcoins are not perishable, a miner's decision on production efforts over the extraction time path is interlinked with its policy over inventory levels. In contrast to a miner that mines a perishable natural resource competitively, a miner in Bitcoin selects a time path for the rate at which it adds to its inventory rather than the rate of extraction, which is exogenous. The reservation demand of miners - or the mined bitcoins that are held in inventory by miners - is influenced, in equilibrium, by the return that their stores generate, which must be equal to the return that the miners can achieve from alternate assets. ${ }^{?}$

So the available supply of bitcoins, $b_{t}$, during period $t$ depends on the coins mined during $t, m_{t}$, and the stock of bitcoins sold by miners from their inventory, $v_{t-1}$. 'Consumption' of bitcoins during $t$ must equal the available supply net of the number of bitcoins that miners hold back in their inventory. Thus, $b_{t}=m_{t}+v_{t-1}$ and $c_{t}=b_{t}-v_{t}$. The inventory level is drawn down if the mining costs incurred between periods exceed the expected return from bitcoin as estimated by miners, $r_{b}$. Thus, the price of the bitcoins held in inventory by the miners must be greater than or equal to the costs they incur to store the coins, $w$, and the rate of return that they expect to receive on their inventory. In other words, for $v_{t}<0$ :

$$
P\left(b_{t}-v_{t}\right)=1 / r_{b} E\left[P\left(v_{t}+m_{t+1}-v_{t+1}\right)\right]-w
$$

The Hotelling growth rule suggests that, within each halving period for Bitcoin, prices would have to rise at least by the rate of interest for miners to be indifferent about whether to increase mining effort or to delay it. The difference between the rate of growth of the spot price and the interest rate modulates mining effort. Note that this is a different consideration for miners than their incentives to deploy costly hashrate in response to extant difficulty levels.

With high capital costs for mining, short-term supply is inelastic, adding a secondary factor to inventory levels, besides planned reservation demand; the more inelastic shorter-term supply is, the more price volatility we ought to expect from changes in demand. On the other hand, there are two countervailing effects that arise on market prices from a natural resource being stored in inventory, especially in the presence of speculative capital. While increasing inventories during periods of declining prices results in price depressions becoming less severe, disposing stocks from inventories curtails price spikes during periods of relative shortages in market supply. Figure 1 illustrates these effects for Bitcoin over the course of a year beginning in March 2020, using the metric of miner rolling inventory (MRI), which exceeds 100 when miners sell from inventories at a faster pace than they mine.

\footnotetext{
${ }^{9}$ To the extent that there are no real alternate uses for dedicated mining rigs, miners can really only engage in mining other proof of work cryptocurrencies to optimise their Hotelling rent.
}

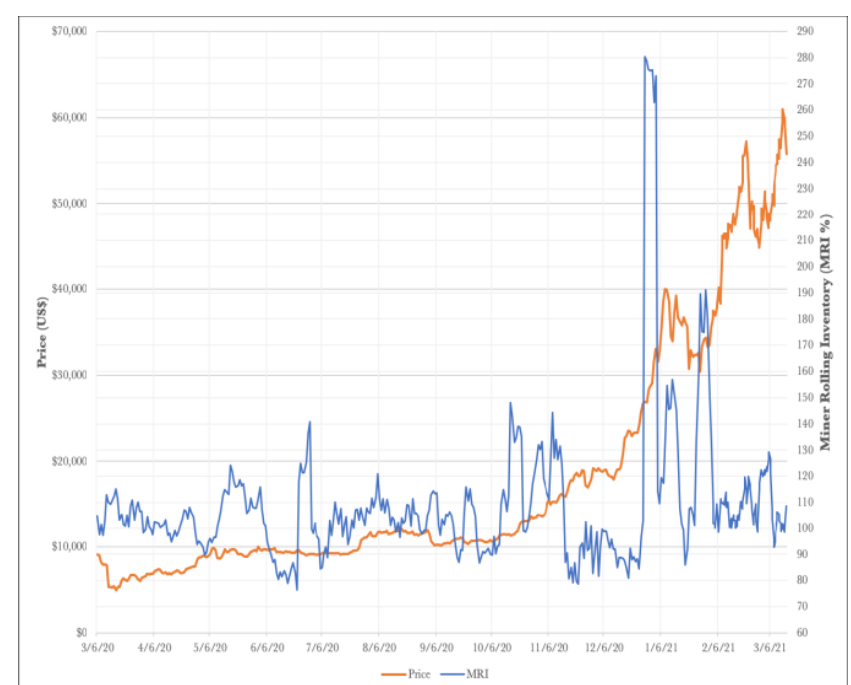

Figure 1: Miner Rolling Inventory and Price (Data Source: Byte'Tree)

In addition to reservation demand by miners, demand for bitcoins is often as a hedge against inflation and systemic risks; an increasing fraction of its consumers, be they individuals or institutional entities, use it as a store of value. As such, the asset is removed from active circulation and becomes the numeraire for measuring intertemporal wealth. Growing longterm inventories have the effect of modulating the Bitcoin market into even more of a traditional exhaustible and nonrenewable scarce resource. In other words, when selling from inventory becomes the predominant source of supply in the market, the optimal time-path of production of traditional exhaustible resources becomes more applicable to the timepath of Bitcoin inventory depletion. For example, as demand becomes more inelastic with high levels of supply-side market concentration, the propensity to sell reduces. An increasing rate of resource depletion over time, coupled with the prospect of decreasing elasticity, requires the rate of growth in price to keep outstripping the discount rate. For a nonperishable good like Bitcoin, this creates a situation for intertemporal arbitrage and increases the marginal propensity to save in earlier periods. Indeed, both these patterns - price growth rates far exceeding the discount rate and a high marginal propensity to save - have been characteristic of the majority of Bitcoin's history.

However, as the stock of a durable good increases, demand must grow at a faster rate than the discount rate for price to increase, regardless of the costs and rate of resource extraction. This can be seen as the minimum threshold for the rate of growth in demand for a stock-to-flow ratio to have a secular effect on price. The price profile has a U-shape for partially durable resources with growth in demand when mining is competitive. [5] Whether the logic applies to Bitcoin depends on whether it can be seen as partially durable. To the extent that the prospect of adverse regulation, elastic rehypothecation, lost wallets and hacked accounts decreases the fully durable characteristic of Bitcoin, this becomes more relevant. 


\section{Modeling the Bitcoin Miner's Inventory Policy}

Given the particular importance of inventories, it is worth considering a stylised model for inventory policy in Bitcoin that underscores the relationship of the policy to optimising capital investments. As a digital resource, mining in Bitcoin depends most critically on the hashing power of the mining 'rigs' and their energy efficiency. Thus, the model gives scenarios for which inventory levels can vary between 0,1 and some fraction between for a given cycle, depending directly on the optimal capital-to-energy ratio.

Assume that at time $t$ a miner uses capital, $K(t)$, and energy, $E(t)$, as inputs in mining Bitcoin, $B(t)$. To simplify the analysis, assume that energy costs grow at a steady rate of $\alpha>0$.

Thus, the rate of bitcoins extracted is given by the miner's production function $B(t)=F(K(t), E(t))$, where $F$ provides constant returns to scale. The miner reserves some of the output as inventory $V(t)$ for investment and sells the rest to the market, $C(t)$, at prevailing prices to cover expenses. Thus, $B(t)=V(t)+C(t)$.

If the share of mined coins reserved for inventory is $v(t) ; v \in[0,1]$, we can write $C(t)=(1-v(t)) B(t)$.

Now assume that the deployed mining rigs become out of date at a rate $\zeta>0$, governed by difficulty adjustments as well as exogenous improvements in technology. Therefore, the growth rate of capital for the miner is given by:

$$
\dot{K}(t)=v(t) B(t)-\zeta(t) K(t) .
$$

In terms of units of energy expended, we can redefine these variables so that we have $\frac{B}{E}=b, \frac{V}{E}=v, \frac{C}{E}=c$ and $\frac{K}{E}=k$. Permitting $f(k)=F(k, e)$ allows us to state Bitcoin's average energy requirement in market consumption, $c(t)=(1-v(t)) f(k(t))$. We assume that $f(k)$ is concave, so that the marginal product of capital increases at a decreasing rate.

Logically, the capital deployment path for the miner depends on both the amount that is invested, in terms of capital's energy requirement, and through considerations over its obsolescence and associated considerations on the availability of energy. Therefore,

$$
\dot{k}(t)=v(t) f(k(t))-(\zeta+\alpha) k(t) .
$$

Crucially, the halving cycles for the block reward plays a key role in Bitcoin. It is, therefore, useful to consider a miner's benefit from a given halving cycle $[0, H]$ as $\int_{0}^{H} c(t) d t$, which then also determines the miner's strategy over inventory, $v(t)$, where $0 \leq t \leq H$.

In other words, the miner's optimisation problem can be stated as,

$$
\operatorname{Max} \int_{0}^{H}(1-v(t)) f(k(t)) d t
$$

$$
\text { subject to } \dot{k}(t)=v(t) f(k(t))-\beta k(t),
$$

where $\beta=(\zeta+\alpha), f_{k}(k)>0$ and $f_{k k}(k)<0$.

Choice over optimal inventory levels, $v^{*} \in[0,1]$, over the halving cycle for the miner is a function of the dynamics of the optimal capital-to-energy ratio, $k^{*}(t)$, over the period. We can assess the trajectory of the optimal inventory level, $v^{*}$, by appealing to the Pontryagin maximum principle $(P M P)$. To do so, we define a function, $g^{*}$, for which we assume $g_{0}^{*}>0$ at $t=0$, such that $\dot{g}^{*}(t)=-g_{0}^{*}\left(1-v^{*}(t)\right) f_{k}\left(k^{*}(t)\right)-g^{*}(t)\left(v^{*}(t) f_{k}\left(k^{*}(t)-\beta\right)\right)$ and $g^{*}(H)=0$.

The $P M P$ then says that the Hamiltonian, $\mathrm{H}$ :

$\mathrm{H}\left(t, k^{*}(t), g^{*}(t), v\right)=(1-v) f\left(k^{*}(t)\right)+g^{*}(t)\left(v f\left(k^{*}(t)\right)-\beta k^{*}(t)\right)$

is maximised by the optimal inventory trajectory.

- As the cycle approaches its completion - i.e. for $t$ nearing $H-g^{*}(t)<1$. With $g^{*}(t)<1 \forall t<t[0, H]$, we would have $\dot{k}^{*}(t)=-\beta k^{*}(t)$ and optimal inventory over the entire halving cycle would just be zero and the optimal capital-to-energy ratio would simply be given by $k(0)^{-e \beta t}$.

- However, it is also possible for there to exist some time, $\hat{t}$, within the cycle where $g *(\hat{t})=1$, while $g^{*}(t)<1$ for $t<H$. For the cases where the optimal inventory is positive, the situation is governed by whether $k$ is above or below the steady-state rate of $\bar{k}$, or the level of $k$ that satisfies $f_{k}(\bar{k})=\beta$. So, if at $t<\hat{t}, g^{*}(t)>1$, it is the case that $k^{*}(\hat{t})$ is less than $\bar{k}$ and then $k(0)$ was less than $\bar{k}$, and we should expect $v^{*}(t)=1$ for all $t<\hat{t}$.

- Between these two extrema for the optimal inventory strategy, over the halving cycle there may be some positive spans of time for which $g^{*}(t)=1$ or, in other words, 
$\dot{g}^{*}(t)=0$. This suggests that $f_{k}\left(k^{*}(t)\right)=\beta$ and $k^{*}(t)=\bar{k}$ for that span of time. In turn, during that time $\dot{k}(t)=0$, the optimal inventory policy is thus given by $0<\beta(\bar{k} / f(\bar{k}))<1$.

The model could be usefully extended to allow for a determinate time path of extraction in Bitcoin, since miners are often faced with additional considerations for their inventory policy. First, miners can readily adjust their optimal capital stock upwards in reaction to a bull market. They cannot, however, reduce capital stock swiftly in a bear market. While inventories can certainly help offset the costs of overcapitilisation in a bull market, they can also build during the bear market in anticipation of the next upward swing in prices and demand. Thus, rather than permitting obsolescence from higher difficulty adjustments in the network, miners are forced to be forward-looking in their inventory policy. Second, to the extent that a determinate extraction path forces the hands of miners in Bitcoin, the option value of investment can come from timing over selecting optimal capital levels (as in [9]) or from simply building up inventories.

\section{Concluding Remarks}

Examining Bitcoin as a digital resource not unlike a traditional natural resource permits us to seek some useful insights from natural resource economics. Indeed, as just another resource, the model presented above ought to seem intuitive, and miners of Bitcoin ought to behave largely like miners of any other physical resource.

The relevance of scarcity of a non-renewable resource to economic growth has largely only been muted on account of positive elasticities of substitution in production or by technological breakthroughs [10]. A contrary logic is applicable to scarce resources that are used as stores of wealth to the extent that they become effective additions to a diversified portfolio. It is particularly worth considering the effects of substitutability between Bitcoin and other physical stores of wealth, such as gold, rare collectibles and real estate. ${ }^{10}$

\section{Competing Interests:}

None declared.

\section{Ethical approval:}

Not applicable.

\section{Author's contribution:}

Prateek Goorha is the main author responsible for writing the manuscript, collecting data, proofreading, etc.
sFunding:

Not applicable.

Acknowledgements:

Not applicable.

\section{References:}

[1] Fantazzini, Dean and Nikita Kolodin (2020) "Does the Hashrate Affect the Bitcoin Price?", Journal of Risk and Financial Management, Vol. 13, No. 11, pp. 263.

[2] Hotelling, Harold (1931) "The Economics of Exhaustible Resources", Journal of Political Economy, Vol. 39, pp. 137-75.

[3] Dai, Min; Wei Jiang; Steven Kou and Cong Qin (2021) "From Hotelling to Nakamoto: The Economics of Bitcoin Mining", NUS RMI Working Paper Series, No. 2021-01.

[4] Landry, Craig E; Dylan Turner and Jeffrey H. Dorfman (2019) "Hotelling Meets Crypto-Currency: Do Bitcoin Rents Follow Hotelling's Rule?”, SSRN Working Paper.

[5] Devarajan, Shantayanan and Anthony C. Fisher (1981) “Hotelling's 'Economics of Exhaustible Resources': Fifty Years Later", Journal of Economic Literature, Vol. 19, No. 1, pp. 65-73.

[6] Goorha, Prateek (2020) "Bitcoinomics 101: principles of the Bitcoin market", Economics Bulletin, Vol. 40, Issue 1, 163-176.

[7] Hayes, Adam S. (2019) "Bitcoin price and its marginal cost of production: support for a fundamental value", Applied Economics Letters, Vol. 26, Issue 7, Pages 554-560.

[8] Levhari, David, and Robert S. Pindyck (1981) “'The Pricing of Durable Exhaustible Resources", The Quarterly Journal of Economics, Vol. 96, Issue. 3, pp. 366-377.

[9] Arrow, Kenneth and Anthony C. Fisher (1974) "Environmental Preservation, Uncertainty and Irreversibility”, Quarterly Journal of Economics, Vol. 88, pp. 312-320.

[10] Solow, Robert M. (2016) "Resources and Economic Growth", The American Economist, Vol. 61, Issue. 1, pp. $52-60$.

[11] Cebrián-Hernández, Ángeles and Enrique JiménezRodríguez (2021) "Modeling of the Bitcoin Volatility through Key Financial Environment Variables: An Application of Conditional Correlation MGARCH Models", Mathematics, Vol. 9, Issue 3, pp. 267.

${ }^{10}$ Most recently, [11] shows uncorrelation between Bitcoin and both gold and oil and correlation with stocks of companies associated with cryptocurrencies and electronic payment systems. 